\title{
MANEJO DE LA FUERZA Y LA VELOCIDAD DEL AGUA: SECTOR ESTE DEL SISTEMA HIDRÁULICO DEL SITIO ARQUEOLÓGICO MONUMENTO NACIONAL GUAYABO DE TURRIALBA
}

\author{
Water force and speed management: East sector of the Guayabo National Monument of \\ Turrialba archeological site hydraulic system.
}

Recibido: $04-07-2020$

Aprobado: 24-07-2020

\author{
María Gabriela Arroyo Wong \\ Universidad de Costa Rica, Costa Rica \\ mariagabriela.arroyo@ucr.ac.cr
}

\begin{abstract}
María Gabriela Arroyo Wong es licenciada en Antropología con énfasis en Arqueología por la Universidad de Costa Rica. Magíster en Gestión Ambiental y Ecoturismo de la Universidad de Costa Rica. Investigadora en temas de arqueología, gestión del patrimonio, incorporación del patrimonio arqueológico al ecoturismo. Docente de la Escuela de Antropología de la Universidad de Costa Rica.
\end{abstract}

\section{RESUMEN}

El sistema hidráulico del sitio arqueológico Monumento Nacional Guayabo se extiende más allá del núcleo arquitectónico donde se ubica el "sistema mayor", posterior a su evacuación, el agua se dirige hacia el este por medio de canalizaciones abiertas, en las cuales es posible encontrar evidencia de estructuras diseñadas para el manejo de la fuerza y velocidad de la misma hasta desembocar en el río Lajitas.

Palabras clave: Monumento Nacional Guayabo; arqueologia; sistema hidráulico; agua; manejo

\section{ABSTRACT}

The hydraulic system of the Guayabo National Monument archeological site extends beyond the architectural core where the "major system" is located, after its evacuation, the water is directed eastward through open pipes, in which it is possible to find evidence of structures designed to manage the force and speed of it until it flows into the Lajitas river.

Keywords: Guayabo National Monument; archaeology; hydraulic system; water; management 


\section{Introducción}

El sitio arqueológico Guayabo de Turrialba se caracteriza por contener gran cantidad de evidencias que reflejan la complejidad social desarrollada, el conocimiento del medio y recursos, así como la implementación de estrategias para su aprovechamiento, entre las que destaca el sistema hidráulico y cada una de las estructuras que lo componen.

Dicho sistema no sólo se ubica en el denominado "núcleo arquitectónico", refiere a un conjunto de estructuras de distinta índole y características que les permitieron a los pobladores el manejo del agua para su total aprovechamiento.

En dirección hacia el este, posterior a la ladera que limita con las estructuras centrales, y hasta el río Lajitas, cruza un cauce que conduce las aguas procedentes del "sistema mayor", y en la época prehispánica también las de la quebrada conocida actualmente como La Chanchera (la cual era direccionada por medio de una canalización abierta ubicada de manera adyacente al norte del montículo central, que posteriormente, alrededor de los años de 1970 fue desviada).

Tomando en cuenta lo anterior, es de esperar que, en el punto de unión de ambos desagües, la cantidad de agua fuera mucho mayor que la que hoy se observa, ya que actualmente es abundante y solamente son las procedentes del "sistema mayor", por lo cual fueron ideadas y construidas una serie de estructuras para controlar la velocidad y fuerza que las caracterizaba.

\section{Estructuras identificadas: "disipador principal"}

Exactamente en el punto donde se unen las canalizaciones procedentes desde el núcleo arquitectónico (desde el "sistema mayor" y el "acueducto abierto"), se localizó una estructura que permite el paso sobre el cauce, a pesar de que este no es profundo la cantidad de agua que pasa es abundante. Para la construcción de la parte superior se utilizaron grandes rocas colocadas unas junto a otras, logrando presión y equilibrio entre ellas, en la parte baja e interna de la estructura se dispusieron rocas más pequeñas, y se colocaron "calzas" para garantizar su permanencia (Figura 1). 
Figura 1. Estructura construida para facilitar el paso y disminuir la fuerza y velocidad del agua procedente del núcleo arquitectónico.

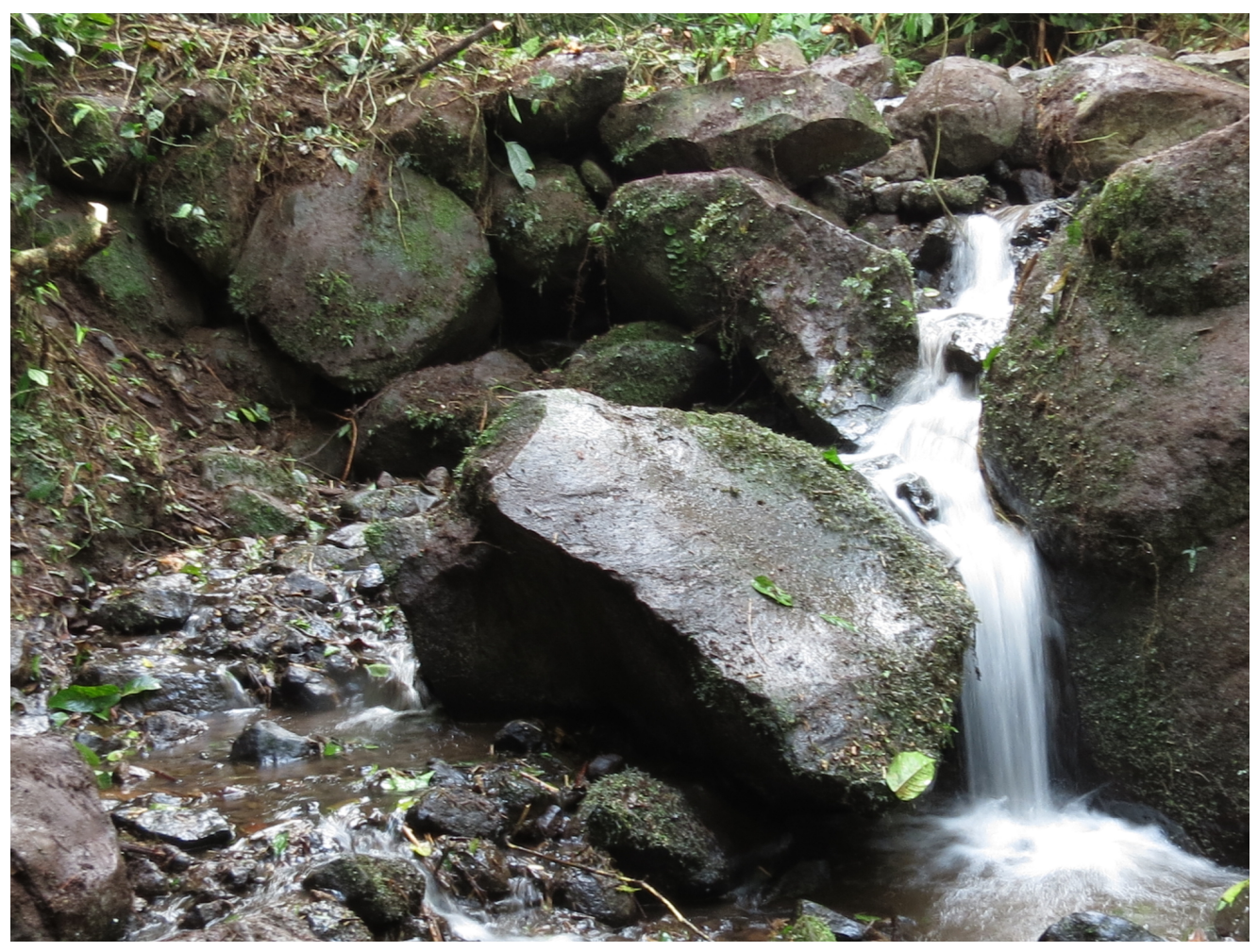

Fotografía: Gabriela Arroyo Wong.

La función disipadora la realiza una gran roca en medio del cauce con otras más pequeñas que hacen que se abarque la totalidad del mismo, el agua pasa por encima de éstas y hace una pequeña caída para seguir por debajo de la siguiente estructura, con la particularidad que esta tiene múltiples "salidas" o "escapes" entre las rocas más pequeñas que se utilizaron en su construcción.

Tomando en cuenta sus características, se le pueden asignar dos funciones, la primera de ellas es la de permitir el paso sobre el caudal y la otra como disipador de fuerza y velocidad del agua (Arroyo, 2016, 2017), este control, les permitió a los pobladores un mayor aprovechamiento del líquido. En las primeras investigaciones realizadas por Aguilar (1972), describió que las escalinatas que se localizan adyacentes, llevaban "hasta una poza muy pintoresca", lo cual refuerza la idea de que posterior a la estructura el agua se mantuviera más en calma. 
Esta estrategia constructiva se repite en el denominado "acueducto abierto" (localizado al norte del montículo central) por el cual fluía la quebrada La Chanchera, el mismo cuando atraviesa el núcleo arquitectónico posee en su parte intermedia un puente y un disipador, es una estructura con la misma función al ubicado en la bifurcación de ambos caudales, con la diferencia de que la segunda es más "masiva", probablemente respondiendo a que capta dos afluentes, lo que se traduce en mayor cantidad de agua.

A nivel estructural, se observan dos diferencias principales, en primer lugar, la ubicada en el núcleo arquitectónico, fue construida de manera que el disipador es independiente al "puente", no obstante, están colocados de manera continua, y la segunda, el paso es facilitado por dos lajas grandes que hacen la función de "puente".

En el caso específico de la estructura ubicada en la bifurcación de las canalizaciones, la misma requirió de otros elementos para darle sostén y fortaleza, en la parte superior se utilizaron grandes rocas colocadas unas junto a otras, de manera que entre ellas mismas se sostuvieran, pero también se colocaron rocas más pequeñas que se introdujeron en la parte baja e interna de la estructura a manera de "calzas" (Arroyo, 2016) (Figura 2).

Figura 2. Estructuras construidas tanto en el sector este como en el núcleo arquitectónico, para facilitar el paso y disminuir la fuerza y velocidad del agua.
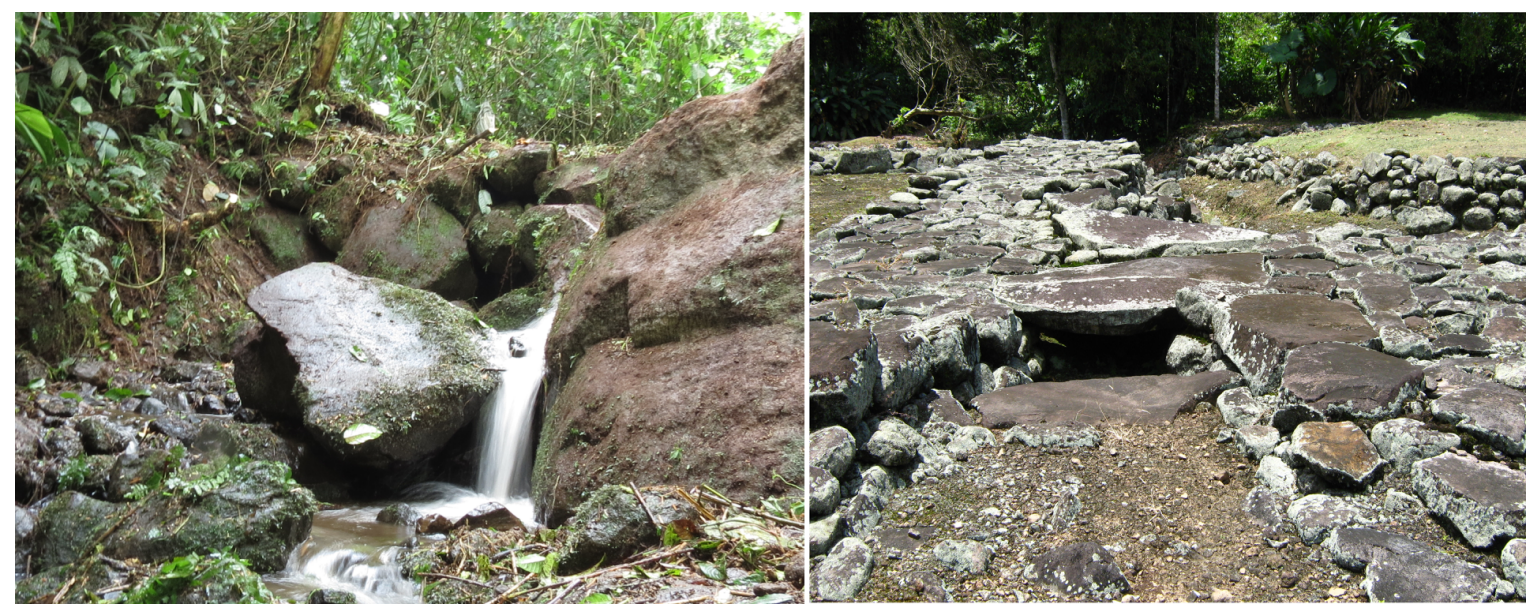

Fotografías: Gabriela Arroyo Wong. 


\section{Disipadores secundarios}

Conforme se avanza sobre el cauce que se dirige al río Lajitas, es posible localizar una serie de "disipadores secundarios", los cuales varían en forma, se les ha consignado este nombre ya que por su disposición y diseño constructivo se logró que la corriente disminuya fuerza y velocidad, de igual manera que con la estructura principal (Arroyo, 2017). A pesar de que realizan la misma función, la diferencia con la primera estructura radica en su tamaño ya que son de menor densidad, esto responde a que desde que se coloca el primer disipador o "disipador principal" ya el caudal ha bajado estos dos factores por lo que rasgos más pequeños son funcionales para dichos objetivos en puntos estratégicos.

Un aspecto importante es que desde el "disipador principal" y entre cada uno de los secundarios la distancia promedio es de $9 \mathrm{~m}$, manteniéndose como una constante. Esto refleja el conocimiento de los pobladores para colocar una estructura cada cierta distancia para lograr un manejo efectivo del recurso.

El primer "disipador secundario", se caracteriza a nivel constructivo por presentar similitudes con el "disipador principal", se aprovecharon dos rocas grandes en la parte superior mientras que en la parte posterior y hacia ambas márgenes se conformaron tres hileras sobrepuestas, entre ellas se colocaron rocas más pequeñas para dar sostén.

En medio de las dos grandes rocas, en la parte superior y media, se colocaron otras pequeñas y medianas, así como lajas, entre ellas mismas se ejercen presión formando una pequeña caída escalonada que termina sobre una roca grande utilizando también la forma natural del cauce. Con esta configuración, se logra canalizar la mayoría del caudal hacia la parte central, obteniendo un mayor volumen y logrando tanto un efecto visual de concentración del mismo, el cual vuelve a disminuir en la caída ya que se expande (Figura 3). 
Figura 3: Primer "disipador secundario", vista de su extremo sur y central.

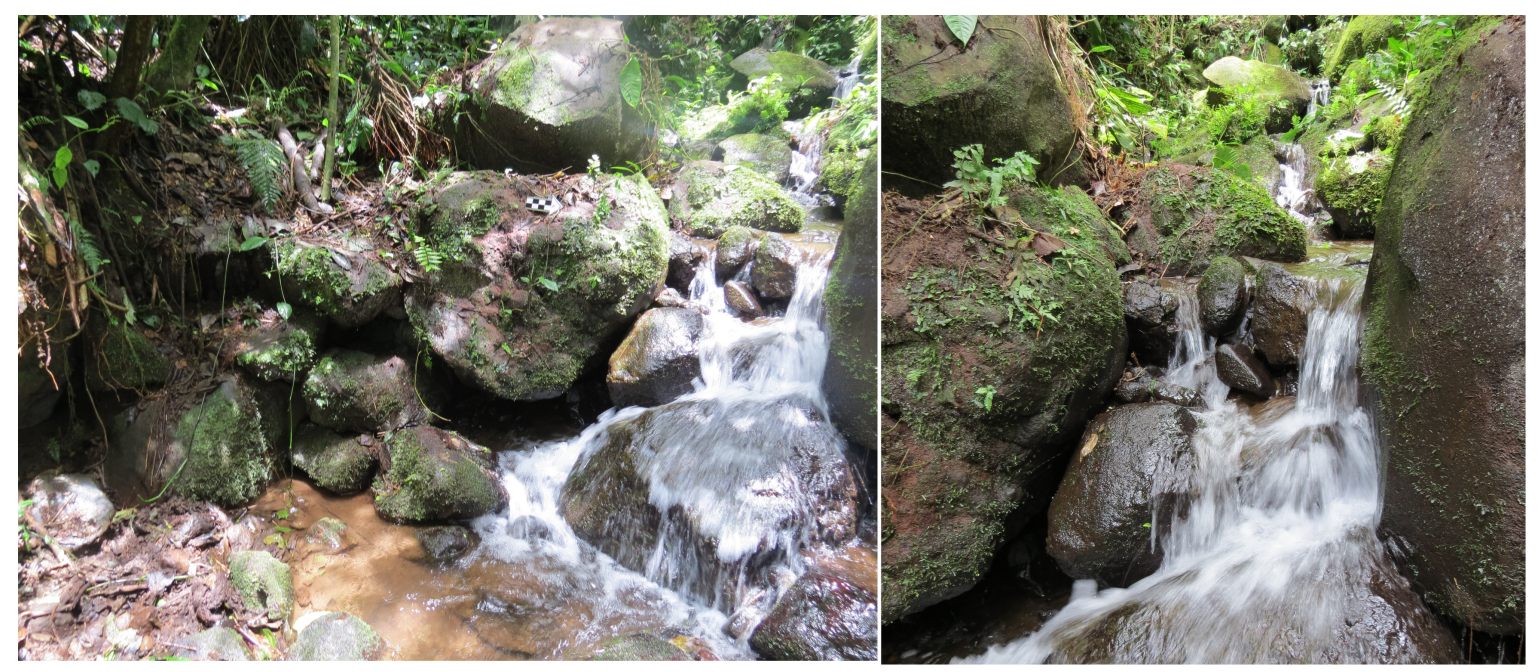

Fotografías: Gabriela Arroyo Wong.

El segundo "disipador secundario", cambia en su construcción, no obstante, supone que una de sus funciones sigue respondiendo a controlar la velocidad y fuerza del agua. Es una estructura más pequeña que presenta como característica una bifurcación marcada por una roca grande, teniendo como resultado dos caídas independientes, se colocaron las rocas de manera que se logra un control sobre el agua direccionándola a ambas salidas.

En la salida de la izquierda, se colocaron de manera intencional rocas pequeñas y fragmentos de lajas calzándose entre ellas, las cuales en su conjunto crean gradientes en diferentes niveles y direcciones, logrando un efecto visual de "zigzag" en su recorrido.

Se da el aprovechamiento de la forma natural del cauce, utilizando una piedra muy plana al final de la caída en "zigzag", lo que provoca que el agua fluya con mayor suavidad. En el caso de la caída de la derecha es sobre una roca colocada de manera perpendicular, al mismo tiempo que se crea una pequeña poza, la cual está delimitada por una roca grande y otras dos más pequeñas que forman una "c", dándole profundidad y límites a la misma (Figura 4). 
Figura 4. Vista general del segundo "disipador secundario", a la izquierda se marca la forma de "zigzag" y aledaña se observa la roca grande que divide el caudal en dos caídas independientes.

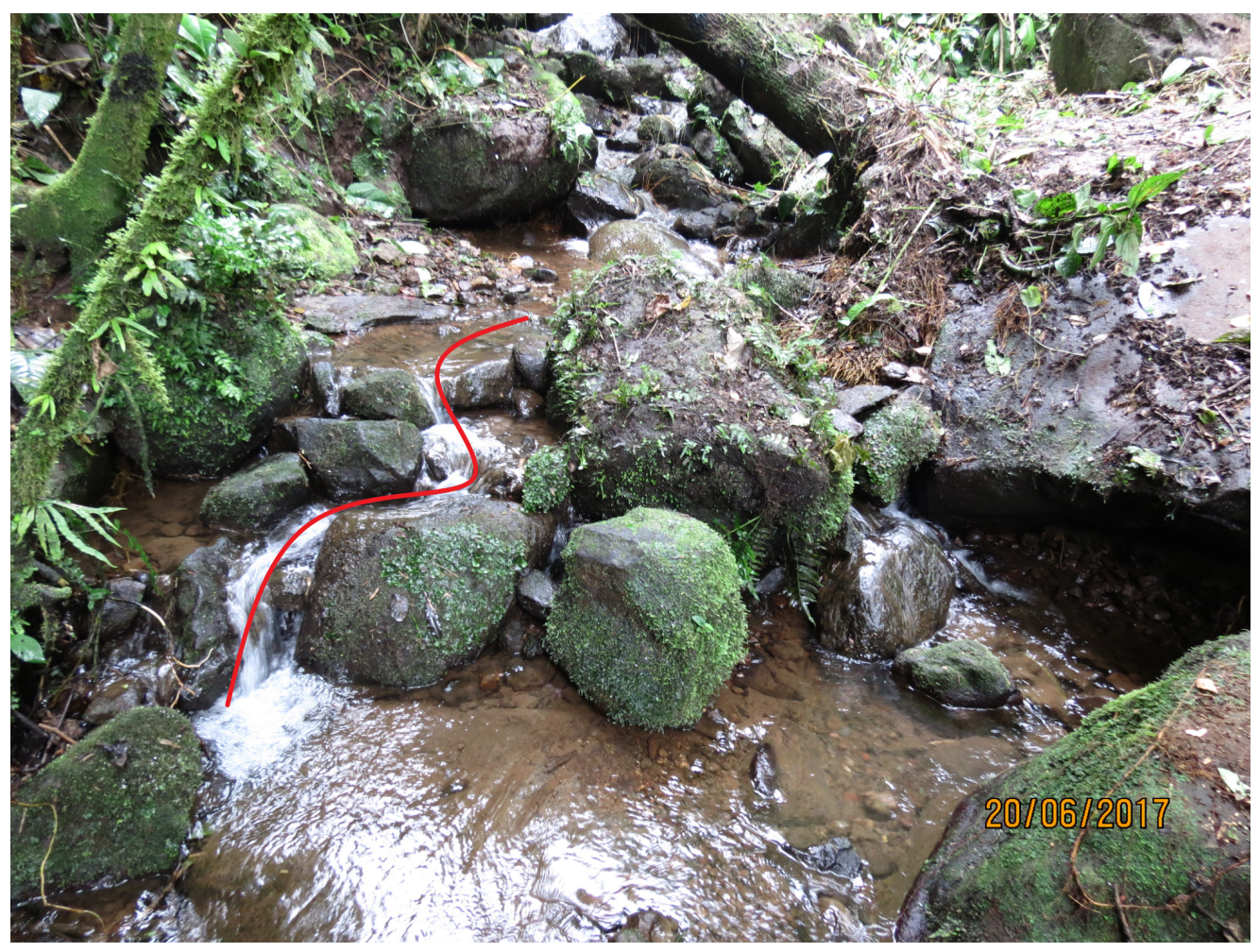

Fotografía: Gabriela Arroyo Wong.

A $5 \mathrm{~m}$ de este rasgo se localizaron tres desniveles formalizados, los cuales simulan gradientes muy sutiles y seguidas entre ellas. Las mismas siguen el curso del caudal conformadas con rocas mediadas sobrepuestas y utilizando más pequeñas como "calzas". Al sur de la última gradiente se ubica un semicírculo construido por medio de tres hileras de rocas sobrepuestas las cuales ejercen presión entre ellas mismas, lo cual hace que se mantengan en esta posición. El agua que se desvía hacia este sector produce un pequeño estancamiento, el agua pasa sobre y entre las rocas que lo conforman (Figura 5). 
Figura 5. Gradientes artificiales que desembocan en una pequeña poza formalizada a través de la colocación de rocas calzadas entre ellas.
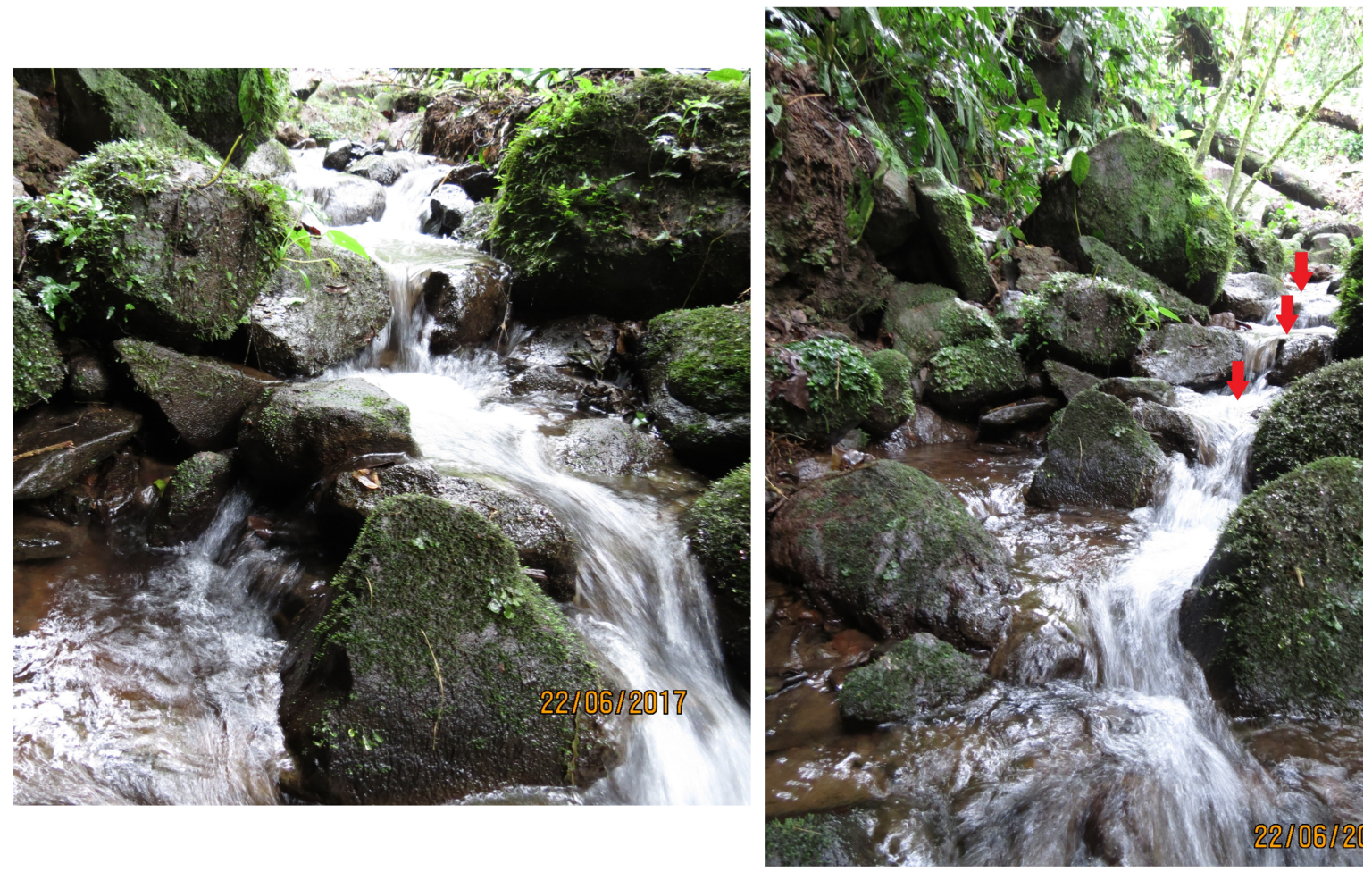

Fotografías: Gabriela Arroyo Wong.

Al otro extremo de este semicírculo el agua pasa bajo una gran roca, la misma desemboca en el que se denominó como el tercer "disipador secundario", el cual se localiza 3 m después de la última gradiente. Este disipador mantiene las características constructivas del anterior con dos salidas formalizadas, la de la izquierda con desniveles y la de la derecha en una caída que desemboca en una poza creada de manera intencional. No obstante, las dimensiones y complejidad de este cambian.

Un elemento de suma importancia en este disipador es la presencia de un petrograbado en el costado sur, el mismo muestra un diseño meádrico y se relaciona directamente con el flujo de agua que discurre a su lado (Figura 6). Este tipo de representación ha sido documentada para otros sitios arqueológicos, un ejemplo de ello es el sitio Rosa María donde Peytrequín y Arce (2016) señalan que predominan los diseños meándricos y las espirales, asociando ambos a rasgos arquitectónicos relacionados con el manejo de aguas, así como al flujo de las mismas. 
Figura 6. Petrograbado asociado al tercer "disipador secundario".
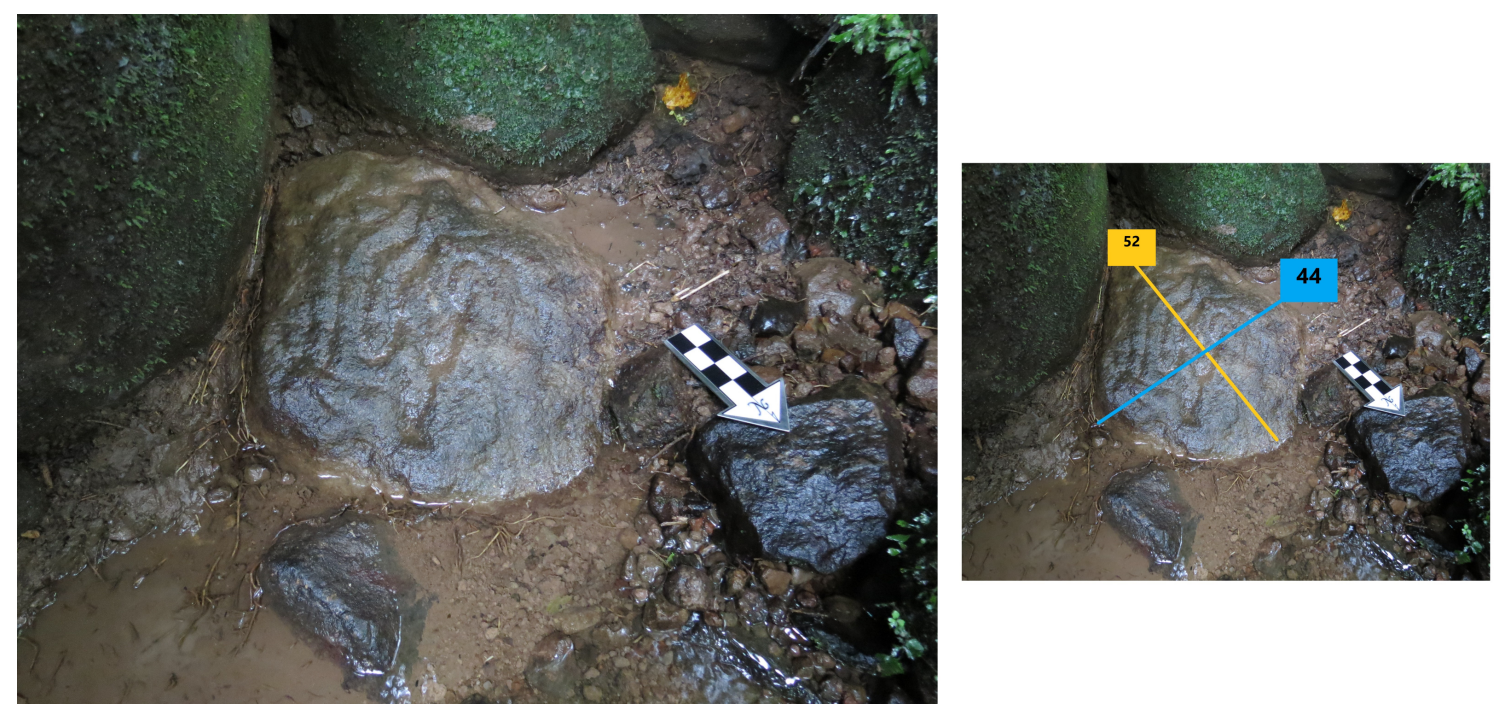

Fotografías: Gabriela Arroyo Wong.

Como se mencionó anteriormente, este disipador se considera más complejo y con una función ampliada con respecto al anterior, ya que, si bien mantiene la estrategia constructiva, las diferencias entre las dos bifurcaciones, las gradientes y el tamaño de la poza son marcadas.

Otro aspecto a resaltar es que a $1.5 \mathrm{~m}$ al este del petrograbado, sobre el cauce y como parte del primer desnivel intencional, entre las rocas que lo constituyen se utilizó una pequeña esfera de $75 \mathrm{~cm}$ de diámetro, la cual está totalmente pulida con evidencia de huellas de trabajo (Figura 7). 
Figura 7. Esfera colocada en la configuración del tercer "disipador secundario".

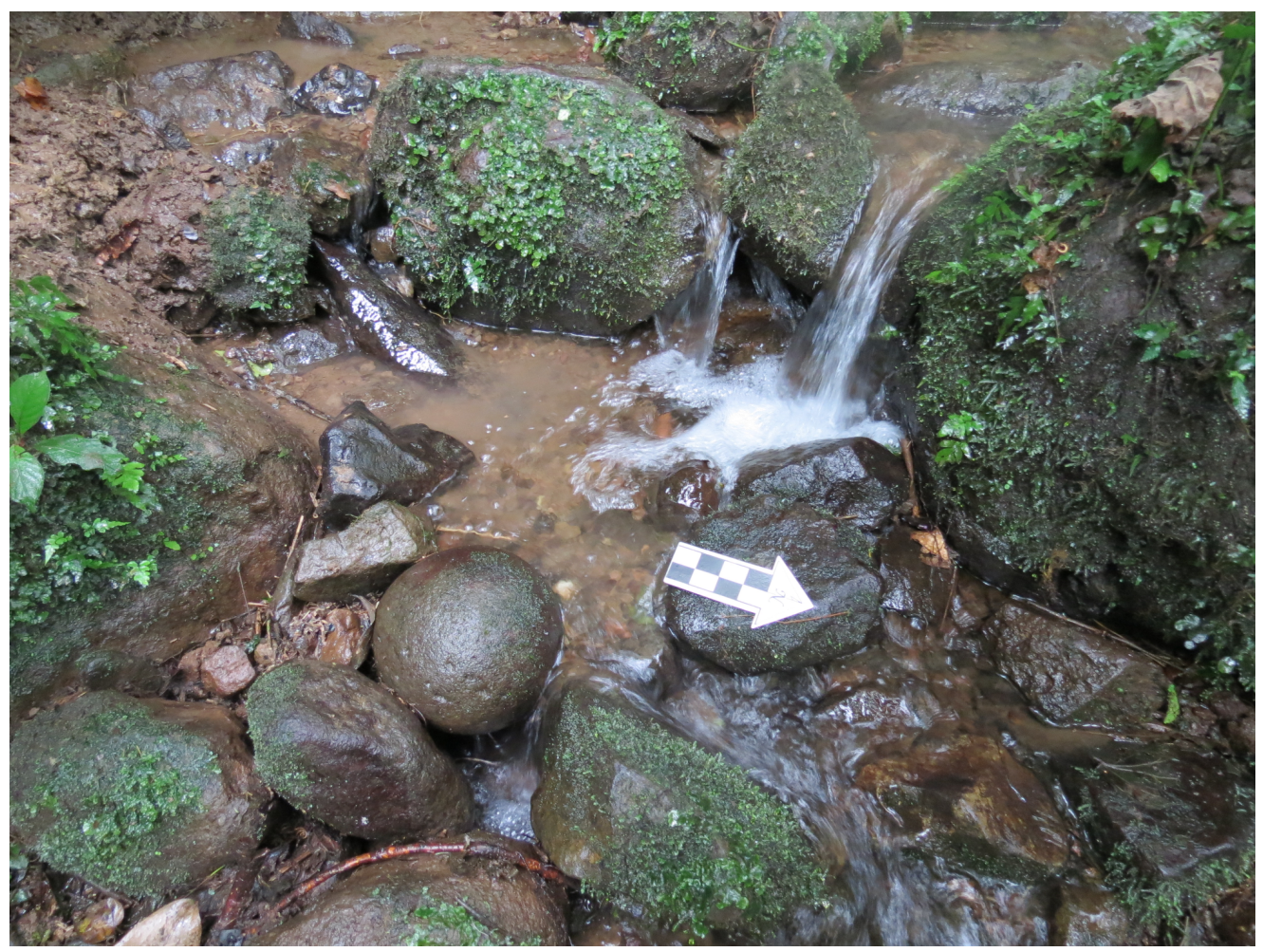

Fotografía: Gabriela Arroyo Wong.

Esta construcción logró no sólo un efecto visual en la manera de correr el agua, también afecta su fuerza y velocidad, en el caso específico del primer desnivel donde se utilizó el elemento esferoidal, el agua debió pasar sobre ella magnificando el efecto visual creado.

No se descarta la posibilidad de que el petrograbado también fue elaborado y dispuesto en una posición en la cual el agua lo cubría en algunos momentos, ya que el caudal era mucho mayor y este se encuentra en el límite actual (Figura 8). 
Figura 8. Ubicación del petrograbado y la esfera.

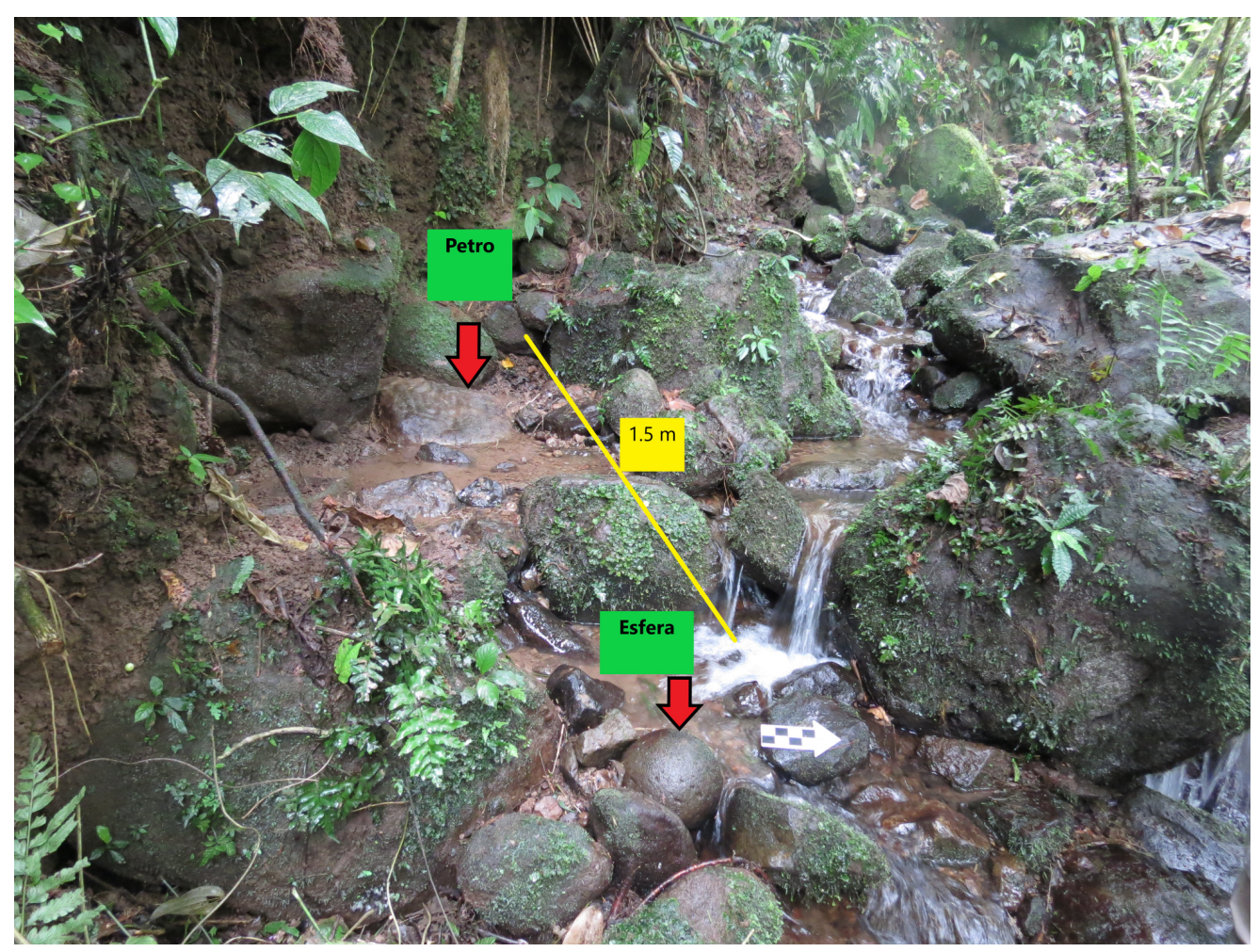

Fotografía: Gabriela Arroyo Wong.

La salida creada a la derecha posee características importantes, una de ellas es la caída que se provoca, la cual es fuerte y evidente a simple vista. El agua que se desvía hacia este sector lo hace por debajo de una roca de gran tamaño, pasando por encima de otra que se dispuso de tal manera que provocara un fluido controlado, a la misma se le fragmentó la parte final (Figura 9).

Más al norte junto a la roca anterior se elaboró otra salida más pequeña que se conforma de varias rocas sobrepuestas de tamaño mediado, una de las cuales presenta un canal de desgaste muy marcado, ambas caídas alimentan la poza ubicada al lado derecho del disipador (Figura 10). Con respecto a esta, posee en su parte central un círculo conformado por rocas grandes y otras más pequeñas, en esta parte se hace más profunda, llegando a $30 \mathrm{~cm}$ y un diámetro aproximado de $60 \mathrm{~cm}$ (Figura 11). 
Figura 9. Roca con fractura intencional.

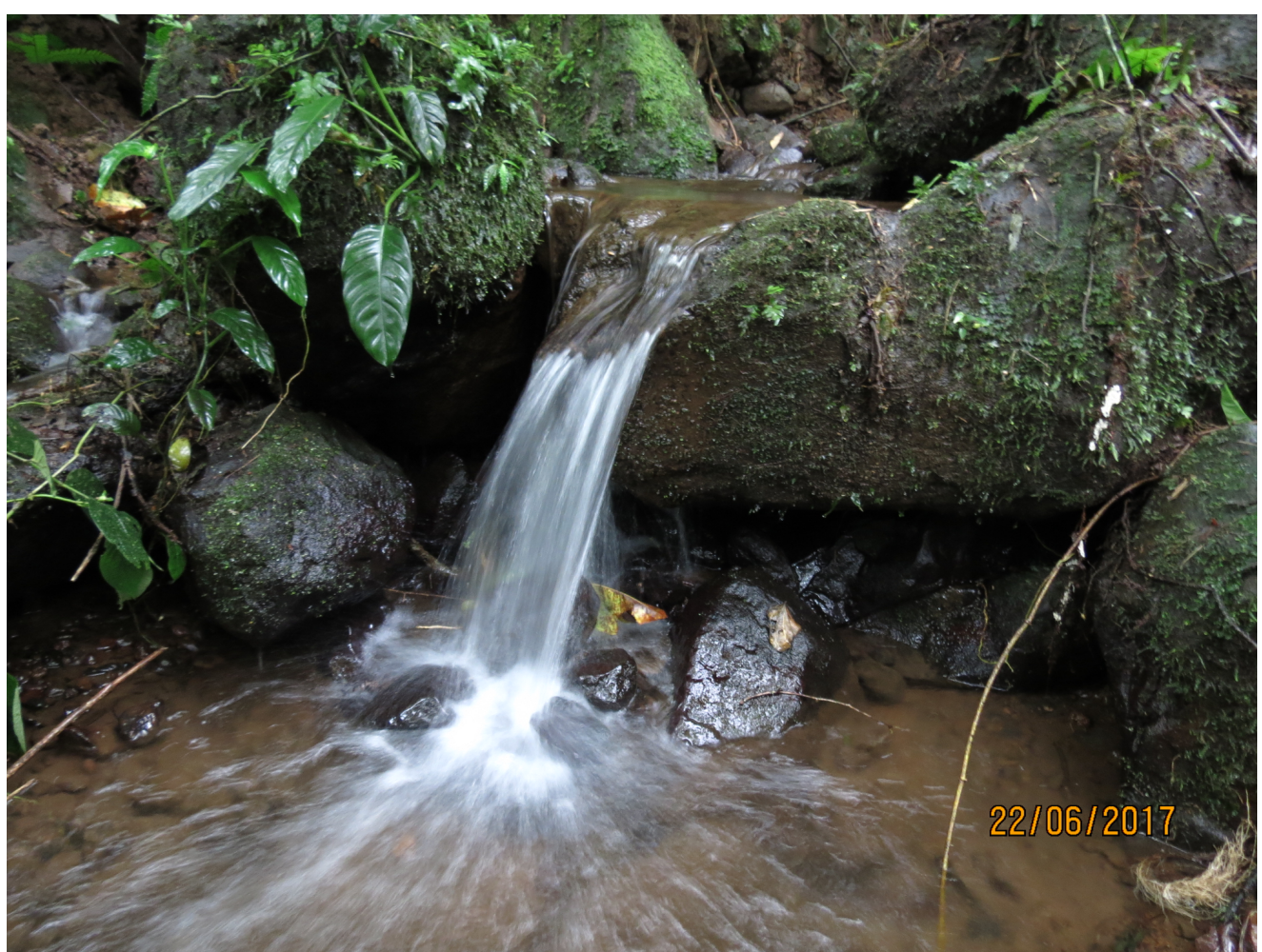

Fotografía: Gabriela Arroyo Wong.

Figura 10. Roca con canal de desgaste.

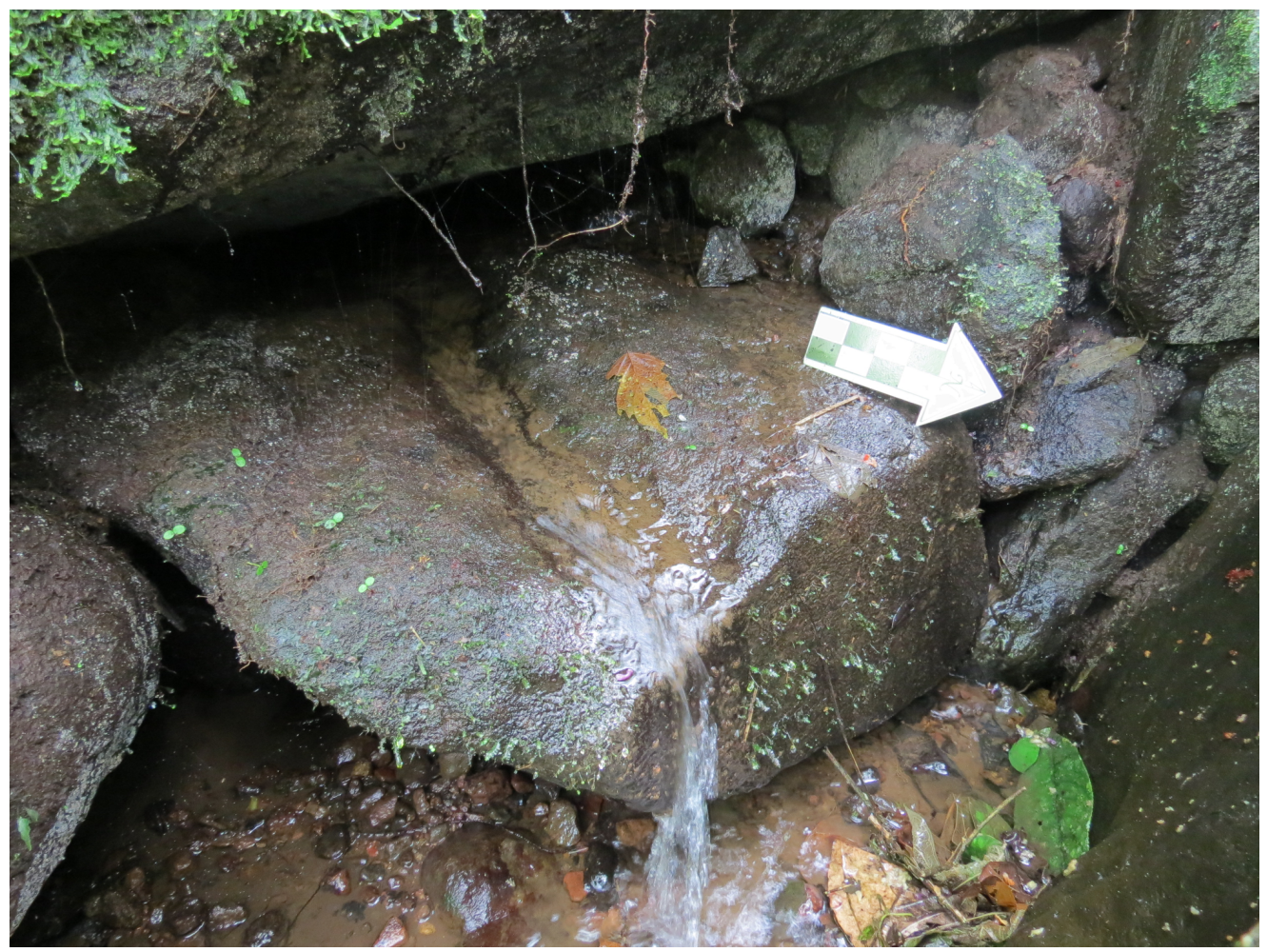

Fotografía: Gabriela Arroyo Wong. 
Figura 11. Poza y círculo conformado en su interior por medio de la colocación de rocas.

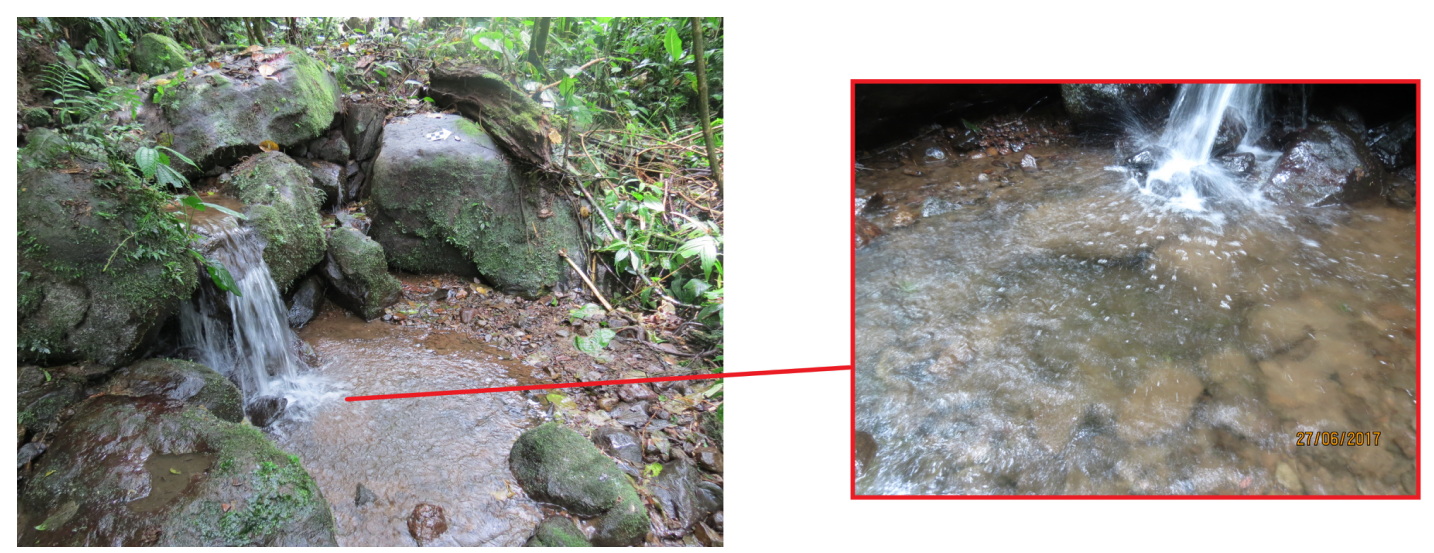

Fotografías: Gabriela Arroyo Wong.

Continuando aguas abajo, después de un pequeño desnivel y de forma casi inmediata se forma otra poza más ancha $(85 \mathrm{~cm})$ pero menos profunda $(15 \mathrm{~cm})$, está alineada con la poza anterior tomando las aguas tanto de allí como las que bajan por la izquierda, a $7.5 \mathrm{~m}$ de esta se encuentra el cuarto "disipador secundario".

Este disipador presenta un primer desnivel sutil conformado por dos hileras sobrepuestas donde se nota la colocación de rocas pequeñas que se calzan entre ellas, está bastante alterado debido a que sobre él yacían dos árboles caídos los cuales le ejercían presión, no obstante parte de la estructura se mantuvo intacta.

En su configuración se colocó un fragmento de metate con su soporte dirigido al este (Figura 12), hacia el río Lajitas, el mismo está entre las rocas que conforman este primer desnivel por lo que las mismas le dan estabilidad ante el paso del agua. Inmediatamente después de esta hilera de rocas, se construyó un muro desde la parte externa del cauce, de dos hileras, pero utilizando rocas más grandes, las cuales se alinean con otra dispuesta al centro del cauce, la cual presenta una concavidad y posteriormente provoca una pequeña caída, formando otra poza de menor tamaño que la del tercer disipador. 
Figura 12. Fragmento de metate utilizado en la configuración del cuarto "disipador secundario".

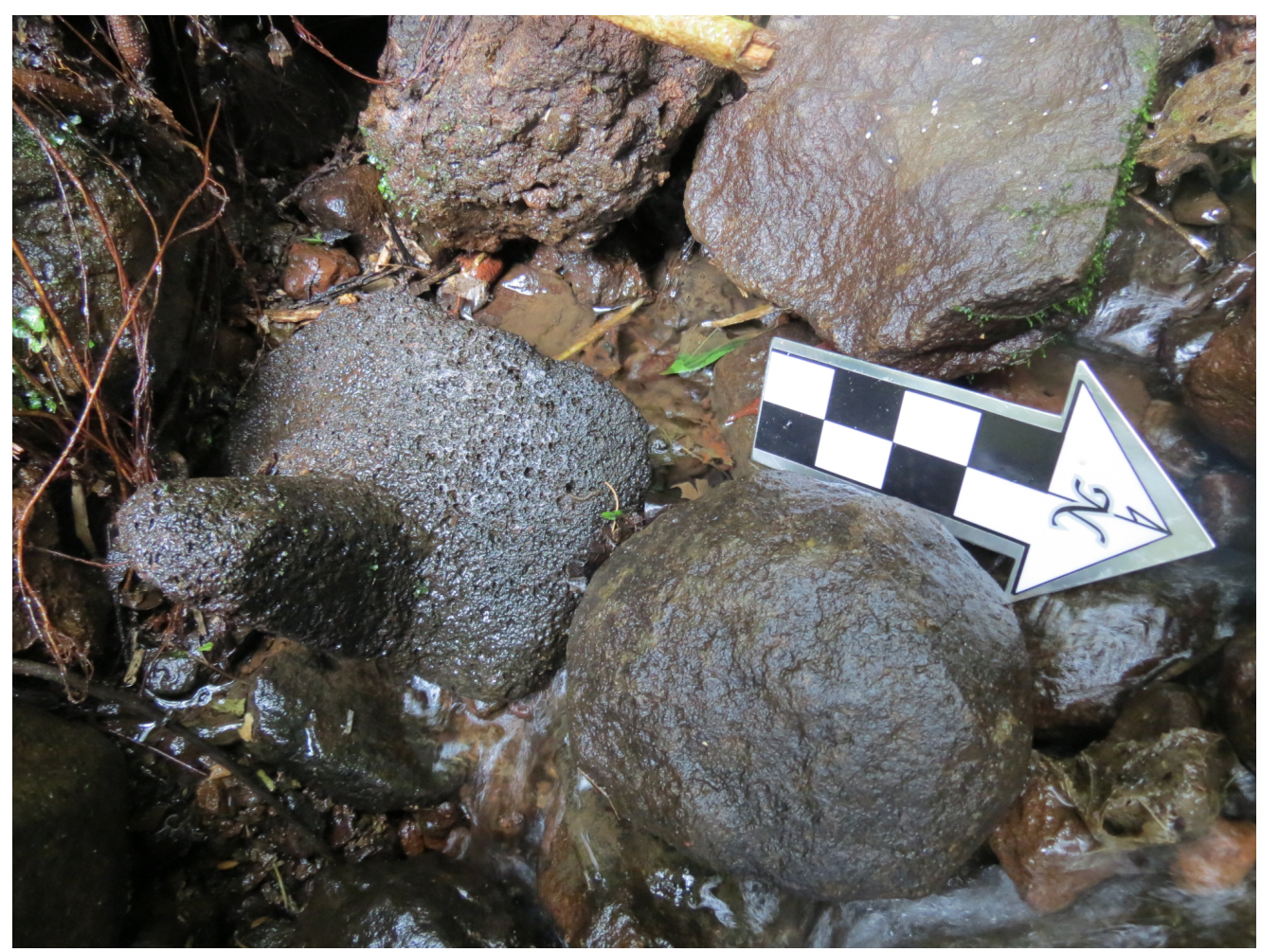

Fotografía: Gabriela Arroyo Wong.

A $10 \mathrm{~m}$ hacia el este del disipador se localizaron dos tumbas de cajón (Arroyo, 2016) a pesar de su relativa cercanía, las mismas están en un nivel más alto que el cauce por lo que no supondría un peligro de ser afectadas.

Continuando el rumbo natural del caudal se identificó el quinto y último "disipador secundario", este parte de una formación natural de rocas de gran tamaño que brindan soporte debido a que en este sector se presenta un desnivel muy marcado.

Posterior a estas rocas, que forman un muro, se dispusieron en su lado sur una serie de rocas angulares y lajas calzadas entre ellas mismas, formando una caída de tres niveles, posterior, se localiza una bifurcación como las registradas para los disipadores anteriores, presentado en su lado izquierdo una caída en varios niveles provocada por la colocación de rocas sobrepuestas y a la derecha la disposición de una sola roca, en este caso en posición horizontal y en el nivel inferior una roca plana. 
Inmediatamente después de la bifurcación realizada de manera intencional, se crean tres pozas pequeñas en "zigzag", lo que provoca una disminución aún mayor de la velocidad y fuerza del agua (Figura 13).

Figura 13. Vista general del quinto "disipador secundario", donde se señalan las dos caídas de agua.

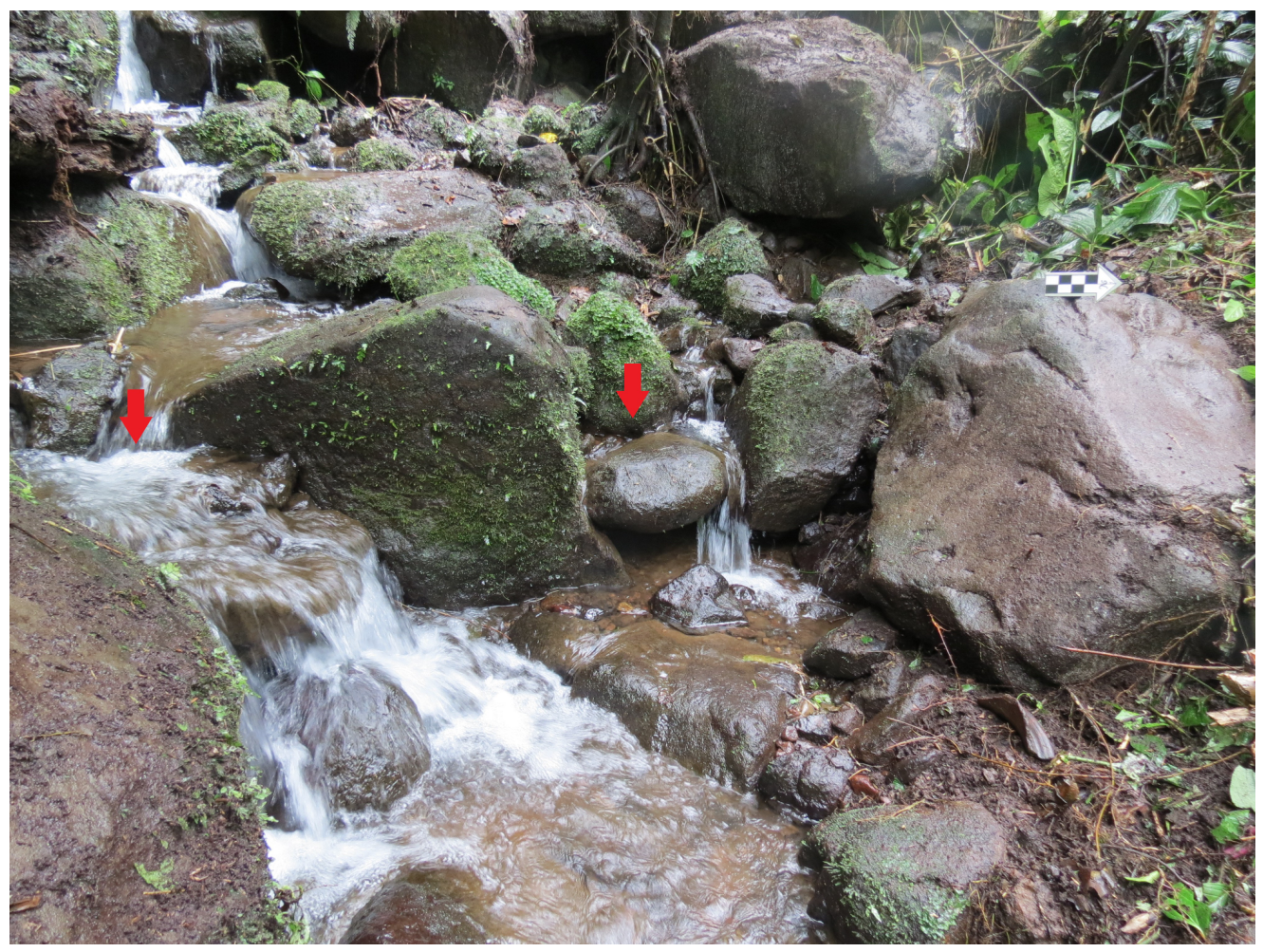

Fotografía: Gabriela Arroyo Wong.

Posterior al último disipador identificado, el caudal hace un viraje sutil hacia el sur, y finalmente se llega al punto donde desemboca al río Lajitas. La salida hacia el afluente mayor se formalizó por medio de la colocación en ambas márgenes de rocas pequeñas sobrepuestas en dos hileras, cabe destacar que el ancho promedio del cauce es de $1.5 \mathrm{~m}$ y en la salida se reduce a $73 \mathrm{~cm}$, casi la mitad.

Hace una caída de $1.6 \mathrm{~m}$ con un ancho de $1 \mathrm{~m}$, la misma es una pared construida con tres rocas grandes al medio, las cuales desde la vista superior se notan dispuestas como "gradas", la inferior más afuera que la superior y llegan a una 
gran roca en el caudal del río Lajitas. Están rodeadas por múltiples rocas pequeñas y lajas calzadas entre ellas mismas, creando un efecto visual muy claro desde el cauce del afluente mayor, desde el cual se puede subir por medio de las tres rocas centrales, pero por la disposición no es posible bajar por ellas hacia el río (Figura 14).

Figura 14. Pared construida con rocas angulares y lajas, que marca la salida de agua procedentes del núcleo arquitectónico hacia el río Lajitas.

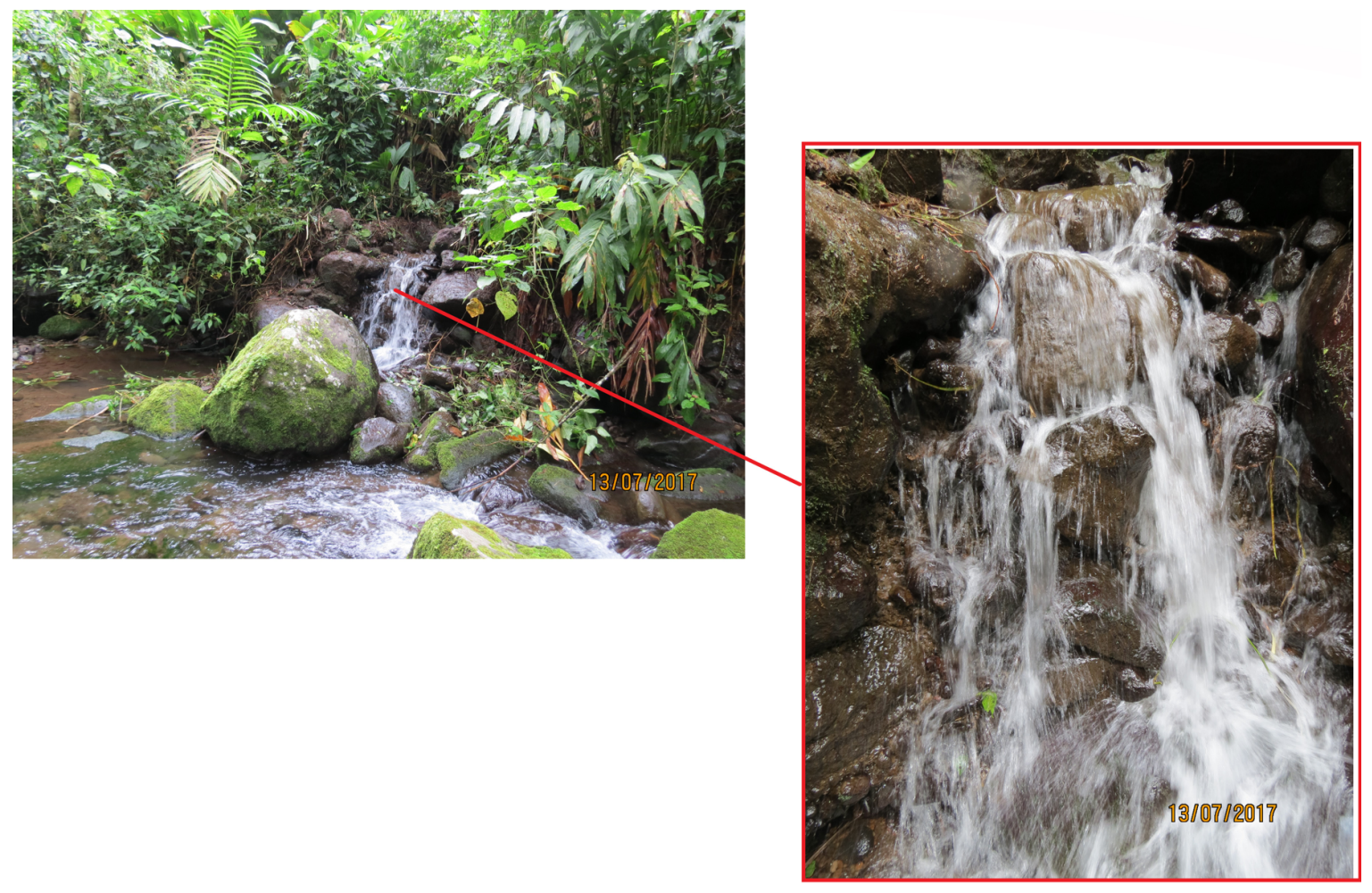

Fotografías: Gabriela Arroyo Wong.

Como se ha descrito, los habitantes del sitio Guayabo, no sólo lograron visualizar y construir estructuras para el manejo del agua en lo que se conoce como el "núcleo arquitectónico", realizaron obras estructurales que les permitió un abordaje que involucra la captación, canalización, distribución, almacenamiento, control y finalmente el direccionar las aguas utilizadas al afluente principal. 


\section{Reflexiones finales}

El sistema hidráulico del sitio arqueológico Monumento Nacional Guayabo, debe considerarse y analizarse en cada una de sus partes, así como relacionarlo directamente a los elementos naturales y culturales que le rodean, ya que se construyó de manera que pasa por diversas áreas con características que requirieron abordajes diferenciados para el aprovechamiento del recurso.

El manejo de las aguas procedentes del núcleo central fue pensado y llevado a cabo por medio de la construcción y disposición de estructuras específicas para el control de la fuerza y la velocidad, se evidencia un conocimiento no sólo del medio y del elemento que se quiere controlar, también desde la parte de ingeniería y arquitectura, dando como resultado un claro aprovechamiento de los mismos para realizar actividades de índole social en torno a ellos.

Lo anterior se refleja en las concentraciones de material lítico y cerámico (principalmente) asociadas a los disipadores, no se descarta la posibilidad de que parte de la evidencia sea producto del arrastre, pero la constante que se presenta es que en cada uno de ellos se registró una densidad mucho mayor.

El registro de este tipo de estructura permite visualizar un sistema complejo, así como una sociedad que aprovechó las formaciones naturales y las complementaron con construcciones ideadas y formalizadas que permitieron no solo el control, sino el máximo aprovechamiento de los recursos.

\section{Bibliografía}

Acuña, V. (1987). Relación entre asentamientos precolombinos al norte de Guayabo de Turrialba en la Fase La Cabaña. Revista de Ciencias Sociales 35, 4352.

Acuña, L., Garnier, E. \& Troyo. E. (1997). Informe de los trabajos de intervención en muro perimetral y escalinata menor del montículo central, Monumento Nacional Guayabo. San José: Ministerio de Cultura Juventud y Deportes. AGORA.

Aguilar, C. (1972). Guayabo de Turrialba. Arqueología de un sitio indígena prehispánico. San José: Editorial Costa Rica. 
Aguilar, M. et al. (2013). Plan de Conservación y Restauración: Monumento Nacional Guayabo 2013 - 2020. San José, Costa Rica.

Alarcón, G. (2015). Configuración del acceso noreste a la aldea precolombina Guayabo de Turrialba (C-362 MNG). Diseño arquitectónico, cronología constructiva y potencial de conservación de la evidencia sobre costumbres alimenticias (219 - B5-079). Informe de temporada 2014 - 2015.

Alarcón, G. (2014a). Informe de labores para el diseño Programa de Investigación Monumento Nacional Guayabo de Turrialba y alrededores 2013-2014 (Documento no publicado). Facultad de Ciencias Sociales, Escuela de Antropología, Universidad de Costa Rica, San José, Costa Rica.

Alarcón, G. (2014b). Estudio de los límites espaciales y temporales del sitio arqueológico Guayabo de Turrialba (C-362 MNG). Segunda fase. Informe temporada 2013.

Alarcón, G. (2013). Informe de labores para el diseño Programa de Investigación Monumento Nacional Guayabo de Turrialba y alrededores 2012 (Documento no publicado). Facultad de Ciencias Sociales, Escuela de Antropología, Universidad de Costa Rica, San José, Costa Rica.

Alarcón, G. (2012a). Informe de labores para el diseño Programa de Investigación Monumento Nacional Guayabo de Turrialba y alrededores 2011 (Documento no publicado). Facultad de Ciencias Sociales, Escuela de Antropología, Universidad de Costa Rica, San José, Costa Rica.

Alarcón, G. (2012b). Estudio de los límites espaciales y temporales del sitio arqueológico Guayabo de Turrialba (C-362 MNG / UCR-43). Informe temporada 2012 (Documento no publicado). Facultad de Ciencias Sociales, Escuela de Antropología, Universidad de Costa Rica, San José, Costa Rica.

Alarcón, G. (2011). Informe de labores para el diseño Programa de Investigación Monumento Nacional Guayabo de Turrialba y alrededores 2010 (Documento no publicado). Facultad de Ciencias Sociales, Escuela de Antropología, Universidad de Costa Rica, San José, Costa Rica. 
Arroyo, G. (2019). Análisis del sistema hidráulico precolombino del Monumento Nacional Guayabo. Informe final. Universidad de Costa Rica.

Arroyo, G. (2018). Caracterización del sector norte, noreste y sureste del sitio arqueológico Guayabo. Ponencia (Documento inédito). Jornadas de Investigación: Inauguración del Centro de Investigaciones Antropológicas (CIAN). Universidad de Costa Rica.

Arroyo, G. (2018). Arquitectura del Monumento Nacional Guayabo: caracterización, ubicación y contextualización. Informe final 2017 - 2018. Universidad de Costa Rica.

Arroyo, G. (2017). Revisión y análisis de los datos derivados de los archivos anteriores e inclusive del año 1985 de los Trabajos Comunales Universitarios del sitio Guayabo de Turrialba del Laboratorio de Arqueología Carlos Aguilar Piedra. Informe final. Universidad de Costa Rica.

Arroyo, G. (2017). Arquitectura del Monumento Nacional Guayabo: caracterización, ubicación y contextualización. Informe parcial de la temporada 2017. Universidad de Costa Rica.

Arroyo, G. (2016). Configuración del acceso noreste a la aldea precolombina Guayabo de Turrialba (C-362 MNG). Diseño arquitectónico, cronología constructiva y potencial de conservación de la evidencia sobre costumbres alimenticias (219 - B5-079). Informe final. Universidad de Costa Rica.

Arroyo, G. \& Arce, M. (2018). Sitio arqueológico Guayabo: nuevos aportes para el análisis del sector norte del núcleo arquitectónico. Ponencia. Simposio 50 años del Laboratorio de Arqueología Carlos Humberto Aguilar Piedra. En prensa. Universidad de Costa Rica.

Badilla, A. (1992). Informe Final Sitio Arqueológico Guayabo: excavación y restauración parcial de la escalinata mayor del Montículo A (Operación 25 Suboperación 2). San José: Ministerio de Cultura, Juventud y Deportes; AGORA S.A.

Baltodano, R., P. Vidal. \& G. Alarcón. (2014). Estudio preliminar de la obra civil en el Monumento Nacional Guayabo, Cartago. Informe de avance de proyecto de investigación. (Documento inédito). Vicerrectoría de Investigación, Universidad de Costa Rica, San José, Costa Rica. 
Barascout C., E \& C Valldeperas. (1992). Plan General de Restauración Monumento Nacional Guayabo de Turrialba. Informe Final. San José: Ministerio de Cultura, Juventud y Deportes; AGORA S.A.

Berrocal, B., J. Chinchilla, \& E. Gutiérrez. (2012). Proyecto: Levantamiento de detalles, curvas de nivel y aforos en la ladera del sector noroeste del Sitio Arqueológico Guayabo. Aporte al proyecto: "Delimitación Espacial del Sitio Arqueológico Guayabo. Evidencia Precolombina de las Modificaciones del Terreno", TC-298 Servicio de Ingeniería a la Comunidad. (Documento no publicado). Vicerrectoría de Acción Social, Universidad de Costa Rica, San José, Costa Rica.

Chinchilla, J.; Mena, V.; \& Tenorio, B. (2016). Uso de tecnologías para el levantamiento y modelado digital de estructuras de interés arqueológico. Documento inédito.

Dubón, J., Solís, H. \& Fonseca, O. (1984). Arqueología e Ingeniería Hidráulica en Guayabo de Turrialba. Primer Seminario Nacional de Ingeniería de los recursos hidráulicos. San José, Costa Rica.

Fonseca, O. (1979). Informe de la primera temporada de re-excavación de Guayabo de Turrialba. Vínculos 5 (1-2): 35-52.

Fonseca, O. \& Hurtdo de Mendoza, L. (1984). Algunos resultados de las investigaciones en la región de Guayabo de Turrialba, Revista de Ciencias Sociales (Edición Especial), 1, 37-51.

Garnier, E. \& Troyo, E. (1995). Informe de los trabajos de excavación y restauración en la escalinata oeste del montículo principal del Monumento Nacional Guayabo de Turrialba-Temporada enero-febrero. San José: Centro de Investigación y Conservación del Patrimonio Cultural; AGORA S.A.

Lange, F. et al. (1987). Principales tipos cerámicos y variedades de la Gran Nicoya. Vínculos 13 (1-2).

León, M. (1986). Análisis funcional de Sitios Arqueológicos en la Zona Protectora Las Tablas, Sur Este de Costa Rica. Vínculos 12 (1-2): 83-120. 
Murillo, M. (2012). Monumento Arqueológico Nacional Guayabo de Turrialba. Su historia, sus investigaciones, su manejo. San José: EUNED.

Murillo, M.; \& Alarcón, G. (2010). Programa de Investigación Monumento Nacional Guayabo de Turrialba y alrededores. Documento inédito. San José: Escuela de Antropología, FCS-UCR.

Peytrequín, J.; \& Arce, M. (2016). Petrograbados y obras hidráulicas precolombinas en el Caribe de Costa Rica. Ponencia para el Congreso Internacional Agua, Cultura y Territorio en Centroamérica. En prensa.

Peytrequín, J.; \& Arce, M. (2015). Obras hidráulicas antiguas del Centro y el Caribe costarricense (600 - 1200 d.C.). Una propuesta formal de clasificación. Hurtado de Mendoza, L. (ed.). Arqueología del Caribe Costarricense. Contribuciones científicas. Volumen 1. San José.

Rojas, R.; Chavez, A.; Guerra, E.; \& Mainieri, O. (1984). Informe semanal del TCU, 30 de enero al 04 de febrero. Universidad de Costa Rica.

Snarskis, M. (1983). La cerámica precolombina en Costa Rica. San José: Instituto Nacional de Seguros de Costa Rica.

Snarskis, M. (1978). The archaeology of the Central Atlantic watershed of Costa Rica. Tesis Doctoral de Philosophy en la Facultad de Ciencias Políticas, Universidad de Columbia.

Snarskis, M. (1976). La Vertiente Atlántica de Costa Rica. Vínculos Vol. 2 № 1, p. 101-114.

Snarskis, M. (1975). Excavaciones estratigráficas en la Vertiente Atlántica de Costa Rica. Vínculos Vol. 1 No 1, p. 2-17. San José.

Torreggiani, I. (2014). Indagine archeologica del sistema idraulico del sito di Guayabo de Turrialba (C-362 MNG), Costa Rica. Scuola di Lettere e Beni Culturali. Universita Di Bologna, Italia. 
Troyo, E. \& E. Garnier. (2002). Acciones para la preservación del sitio Guayabo: consolidación y restauración. Guayabo de Turrialba: Una aldea prehispánica compleja. Ministerio de Cultura, Juventud y Deportes, Centro de Investigación y Conservación del Patrimonio Cultural. San José, Costa Rica; 71-135.

Vázquez, R. (2014). Turrialba, una encrucijada: arqueología regional y rutas antiguas de comunicación en un valle del trópico húmedo de Costa Rica (11.000 a.C.-1600 d.C.). Tesis de Doctorado, Department of Anthropology, University at Albany, State University of New York.

Vázquez, R. (Coord. y Ed.). (2002). Arqueología del área de influencia del Proyecto Hidroeléctrico Angostura, Valle de Turrialba. Convenio ICE-MNCR (Documento no publicado), Instituto Costarricense de Electricidad, Museo Nacional de Costa Rica, San José, Costa Rica.

Vázquez, R., H. Massey, \& J. C. Sánchez. (2002). Guayabo y su relación con el valle de Turrialba en el Período VI (1000-450 a.p.): prospección de las calzadas Caragra y Alto Varas. En Arqueología del área de influencia del Proyecto Hidroeléctrico Angostura, valle de Turrialba. Vázquez, R. (ed.). Informe, Convenio Instituto Costarricense de Electricidad-Museo Nacional de Costa Rica, San José. p. 315-333. 